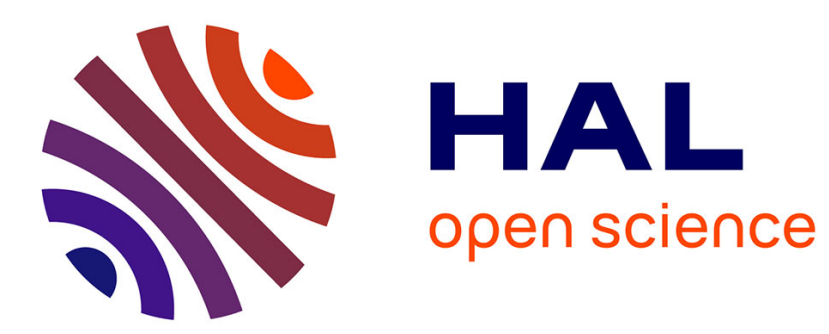

\title{
A neural network approach for the development of modular product architectures
}

\author{
John Pandremenos, George Chryssolouris
}

\section{To cite this version:}

John Pandremenos, George Chryssolouris. A neural network approach for the development of modular product architectures. International Journal of Computer Integrated Manufacturing, 2011, 10.1080/0951192X.2011.602361 . hal-00732119

\section{HAL Id: hal-00732119 \\ https://hal.science/hal-00732119}

Submitted on 14 Sep 2012

HAL is a multi-disciplinary open access archive for the deposit and dissemination of scientific research documents, whether they are published or not. The documents may come from teaching and research institutions in France or abroad, or from public or private research centers.
L'archive ouverte pluridisciplinaire HAL, est destinée au dépôt et à la diffusion de documents scientifiques de niveau recherche, publiés ou non, émanant des établissements d'enseignement et de recherche français ou étrangers, des laboratoires publics ou privés. 


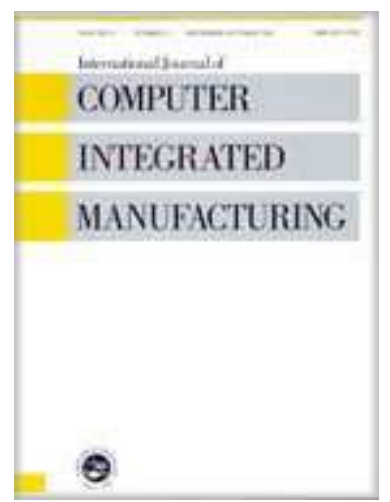

\section{A neural network approach for the development of modular product architectures}

\begin{tabular}{|c|c|}
\hline Journal: & International Journal of Computer Integrated Manufacturing \\
\hline Manuscript ID: & TCIM-2010-IJCIM-0041.R1 \\
\hline Manuscript Type: & Original Manuscript \\
\hline $\begin{array}{r}\text { Date Submitted by the } \\
\text { Author: }\end{array}$ & $19-N o v-2010$ \\
\hline Complete List of Authors: & $\begin{array}{l}\text { Pandremenos, John; University of Patras, Laboratory for } \\
\text { Manufacturing Systems and Automation } \\
\text { Chryssolouris, George; University of Patras, Laboratory for } \\
\text { Manufacturing Systems and Automation }\end{array}$ \\
\hline Keywords: & DESIGN, NEURAL NETWORKS \\
\hline Keywords (user): & DSM \\
\hline
\end{tabular}

\section{SCHOLARONE ${ }^{\text {M }}$ \\ Manuscripts}




\title{
A neural network approach for the development of modular product architectures
}

\section{J. Pandremenos and G. Chryssolouris*}

Laboratory for Manufacturing Systems and Automation, University of Patras, Rio, Patras 26500, Greece

*Corresponding author.

Tel. +302610997262

Fax. +302610997744

Email: xrisol@1ms.mech.upatras.gr

\begin{abstract}
The clustering of a product's components into modules is an effective means of creating modular architectures. This paper initially links the clustering efficiency with the interactions of a product's components and interesting observations are extracted. A novel clustering method utilizing Neural Network algorithms and Design Structure Matrices (DSMs) is then introduced. The method is capable of reorganizing the components of a product in clusters, in order for the interactions to be maximized inside and minimized outside the clusters. Additionally, a multi-criteria decision making approach is used, in order for the efficiency of the different clustering alternatives, derived by the network, to be evaluated. Finally, a case study is presented to demonstrate and assess the application of the method. The derived algorithmic clustering proved to be more efficient compared with the empirical one and thus, it can be used by design engineers as an effective tool for the derivation of product clustering alternatives.
\end{abstract}

Keywords: design; Neural Networks; DSM

\section{Introduction}

A major customer demand is often the tailoring of a product to specific, individual

needs. The challenge is how to design and produce fast and inexpensively,

Deleted: high quality

customized and easily adaptable goods of a high quality that are adequate for a mass

market. The development of modular design architectures is considered as an

efficient practice in order for this challenge to be met. The clustering of a product's

components into modules is such an approach that involves the procedure of grouping

Deleted: which

these components together, in order for dense interactions to be maintained within the

cluster and sparse outside them. The clustering technique transforms a non modular,

at part level, product design architecture into a modular one, at a clusters level. 
A tool often used for the representation of the interaction among the elements of a system, whether the system is a product, process or organization, is the Design Structure Matrix (DSM). These matrices are usually binary, square and contain the name of the system's elements along their side (as row headings) and across the top (as column headings). Whilst a link exists between node $c$ to node $d$, the value of the $c$ - $d$ element is regarded as unity or it is marked with $\mathrm{X}$, otherwise, the element value is zero or it is left empty. The diagonal elements of such matrices usually have a zero value or they are left empty as well, since they do not play any role within the matrix (Ulrich and Eppinger 2003). Clustering in a DSM is accomplished when the elements of the matrix are rearranged so as to form groups, which comply with the clustering rule: maximum interactions within the clusters and minimum outside them.

Table 1 shows examples of different clustering types.

$\underline{\text { In literature, a large amount of efficient methods for the development of }}$ modular design architectures is available: from the Axiomatic Design Theory of Suh (1990) and the Total Design of Pugh (1991) to the adaptable design of Gu et al. (2004), the Modular Function Deployment of Ericsson and Erixon (1999) and the Platform-Based Product family Development of Simpson et al. (2006). However, for the purposes of this paper, only approaches focusing on the DSM clustering are reviewed.

A large number of the clustering algorithms developed, are based on Genetic Algorithms (GAs). Yu et al. $(2003,2007)$ developed a GA in order to perform DSM clustering, using as criterion the minimum description length (MDL) principle that is based on information theory. Some interesting features of their algorithm are the possibility of having overlaps among the modules as well as the capability of performing bus clustering. In 2006, the NASA Scientific and Technical Information 
(STI) Program Office developed in Excel ${ }_{2}$ an efficient GA that was performing clustering on DSMs included in a spreadsheet format within the program (Rogers $e t$ al. 2006). Idicula's (1995) approach was the first successful attempt to perform clustering with a stochastic algorithm that was attempting iteratively to decrease the value of a total coordination cost function. A couple of works were based on this approach and developed it even further (Fernandez 1998, Thebeau 2001).

Mueller (2004) employed the King's and the Modified Minimum Degree algorithms so as to perform a sparse matrix reordering ${ }_{2}$ in dot plot visualizations, to reveal clusters within a data set. Dot plots, similarly to DSMs, are used to displaying relationships among the elements in a data set. Sangal et al. (2005) represented complex software architectures with DSMs and attempted to manage them with the help of Lattix Inc's Dependency Manager (LDM). LDM is the first commercially available implementation of the DSM analysis for software. It offers algorithms that may perform partitioning of DSMs, a task which is quite similar to clustering.

Browning (2001), in his review work, explained in detail, the importance of the DSM clustering, described its different types, presented a number of algorithms developed for that reason and gave examples of their application. Yassine and Braha (2003) showed how clustering of project tasks with the help of DSM representations can facilitate the concept of concurrent engineering. Furthermore, Yassine (2004) employed DSMs and clustering algorithms for the planning, execution and management of complex product development projects. Several clustering algorithms that utilize simple mathematical operations have been developed for the formation of "families" in process planning (Chryssolouris 2006). Additionally, Oliveira et al. (2009) proposed a bipartite graph modelling with a graph clustering algorithm for the partitioning of parts into "families" and the machines of a shop floor into groups, in 
order to obtain manufacturing cells. Finally, Vesanto and Alhoniemi (2000) investigated different approaches for the clustering of Self-Organizing Maps (tool for data mining). Special attention was given to agglomerative clustering and partitive clustering using k-means.

The above literature review has revealed that few techniques for the clustering of a product parts there exist. A major part of these methods, which are indeed the most efficient ones, is based on GAs in conjunction with the DSMs. Moreover, the survey has shown that currently, there is no algorithm enabling the designer to preselect the number of clusters that will be deriving from the clustering procedure.

The objective of this work is to investigate the clustering efficiency of the different design architectures (at part level) as well as to develop a method, capable of performing the clustering of a product's components by using DSMs and Neural Networks. In the next section, the clustering efficiency of the different architectures is assessed. Section 3, describes comprehensively the clustering method proposed. Section 4, examines the application of the new method to a real case study, a "state of the art" car "Body in White". In the last section ${ }_{2}$ the results derived are evaluated and conclusions are drawn.

\section{Clustering Efficiency Assessment}

Two main architectures are recognised in design, the integral (coupled interactions among parts) and the modular one (uncoupled interactions between parts) (Ulrich 1995). However, very often, products of combined architectures exist.

The efficiency of a product's components clustering is directly related to the product's design architecture, at part level. A product, having the fewest possible interactions among its parts, is considered being an ideal modular product, at part 
level. The clustering of the parts of such a product would result in sparse clusters, also with sparse interactions among them (Figure 1a). On the other hand, an ideal integral product, at part level, has the maximum possible number of interactions and therefore, the application of the clustering technique to this product would lead to dense clusters with dense interactions among them (Figure 1b).

Through the aforementioned observations and by going over the definition of the ideal clustering (dense interactions within the clusters and sparse among them) it may be concluded that clustering is not worthy when having ideal design architectures (either integral or modular). In the case of a combined architecture (nowadays trend), efficient clustering is feasible as it can be seen in the example of Figure 1c. The clustering technique transforms non modular product design architecture into a modular one.

Pandremenos et al. (2009) have developed an index for the quantification of the design architecture of a product, at part level. The index was called "Modularity Performance" $(M P)$ and was given by the following equation:

$$
M P=1-\frac{I-I_{\min }}{I_{\max }-I_{\min }}
$$

Where $I$ the number of interactions within the parts of a product, $I_{\max }=\left(n^{2}-\right.$ $n) / 2$ and $I_{\min }=n-1$, where $n$ the number of parts. $M P$ derives from the normalization of $I_{\max }$ and $I_{\min }$ values in order for the same boundaries, between 0 and $1\left(I_{\max }\right.$ and $I_{\min }$ values depend on $n$ and therefore, are not constant) to be always maintained.

Obviously, when $I=I_{\max }, M P=O$ (Integral architecture) and when $I=I_{\min }, M P=1$ (Modular architecture).

This index could be employed by designers so as to assess the design architecture of a product and thus, decide if the clustering of this product is worthy or 
not. The closer the $M P$ value would be to the boundaries ( 0 or 1$)$ the less worthy would be the clustering of this product.

\section{DSM Clustering Algorithm}

The problem of clustering the components of a product into modules, in terms of their interactions, is non linear. Neural Networks are well known for their capability of solving non linear problems. The algorithm proposed in this paper, is based on Self

Organizing Neural Networks (SONNs) trained with unsupervised competitive learning and on DSM representations. The idea is to represent the interactions of each product's components through the DSM, with vectors, which are afterwards inserted into the network for clustering. This may be the first attempt for solving the DSM clustering problem with such an approach.

\section{1. $\quad$ Algorithm description}

The SONNs are widely utilized for the clustering of multidimensional data. The layout of such a network consists of three layers: the input, the competitive and the output (Figure 2). A brief explanation of each layer follows:

- Each node $\left(v_{\boldsymbol{l}} \ldots \boldsymbol{v}_{\boldsymbol{i}}\right)$ of the input layer is a $n$-dimensional vector.

- Each node of the competitive layer is a neuron as in Figure 3. The number of neurons is equal to that of the desired clusters to be produced.

- The single node of the output layer indicates the cluster each element of the input layer belongs to.

The main idea behind the SONN is to group together the elements of the input layer.

Each neuron (Figure 3) of the competitive layer is composed of:

- Weights $\left(\boldsymbol{w}_{j}\right)$ 
- These weights are vectors of the same dimension $(n)$ as the input data vectors. The distance between $\boldsymbol{v}_{i}$ and $\boldsymbol{w}_{j}$ is the main criterion of the $j^{\text {th }}$ neuron for selecting or not, the $i^{\text {th }}$ element for the $j^{\text {th }}$ cluster.

- Biases $\left(\boldsymbol{b}_{j}\right)$

The role of the bias is very important. The bias either "helps" to or "prevents" the elements from being grouped in certain clusters. It is formed in such a way so as to secure the formation of a predefined clusters' number of about the same number of elements. Consequently, the absence of bias would permit the network to form fewer clusters than the desired number and in a different magnitude.

- A distance function (DIST)

It calculates the Euclidian distance between $\boldsymbol{v}_{\boldsymbol{i}}$ and $\boldsymbol{w}_{j}$.

- A summing function It adds the distance to the bias.

- A competitive transfer function $(C)$

It decides whether or not the $i^{\text {th }}$ element will belong to the $j^{\text {th }}$ cluster.

At each run of the network, the input $v_{i}$ enters one after the other all the neurons (cluster) and the Euclidean distance between $\boldsymbol{v}_{i}$ and $\boldsymbol{w}_{j}$ is calculated. Then, this calculated distance is added to the bias of the neuron in the summing function. Finally, the sum enters the competitive function $(C)$, which decides whether or not the $v_{i}$ is going to be clustered into the certain cluster-neuron (Figure 3). The initial value given to the weights occurs from the midpoint function (places the weights in the middle of the input ranges). The cluster-neuron that wins the input $\boldsymbol{v}_{i}$ is called the "winner" neuron. After the completion of each run, the network provides a clustering result. However, in order for the network to get trained, the aforementioned procedure is iterated (epochs). At each epoch, the weights are updated based on the Kohonen learning rule and the biases, according to the "learncon" learning function (it grows the bias disproportionally to the percentage of the wins that a neuron accomplishes). Three hundred epochs are sufficient for a well trained network (Kohonen 2001).

\subsubsection{Example}


The 2-dimensional data are the simplest to cluster with the SONNs. A matrix representation of these data, which comprises the input vectors $v_{i}$ in the network, is shown in the following equation. In this matrix, the index $i$ is the number of input data, $\boldsymbol{i}$ and $\boldsymbol{j}$ are the co-directional unit vectors to axis $x\left(1^{\text {st }}\right.$ dimension $)$ and $y\left(2^{\text {nd }}\right.$ dimension) respectively, and finally, $x_{i}$ and $y_{i}$ are the lengths of $\boldsymbol{v}_{i}$ at each dimension.

$$
\left[\begin{array}{llll}
v_{1} & v_{2} & \cdots & v_{i}
\end{array}\right]=\left[\begin{array}{cc}
x_{1} & y_{1} \\
x_{2} & y_{2} \\
\cdot & \cdot \\
x_{i} & y_{i}
\end{array}\right]\left[\begin{array}{l}
\vec{\imath} \\
\vec{\jmath}
\end{array}\right]
$$

Assuming that we have the $i=100$ input data which we intend to cluster into three modules, shown in Figure 4-left. These data were deliberately selected to form three point clouds, in order to be demonstrated that the three clusters are correctly defined by the network. In order to obtain this amount of clusters, the network was set to consist of three neurons, in the competitive layer and therefore, also three weights exist, one for each neuron $(j=3)$. All the weights $\boldsymbol{w}_{j}$ in their initial position were located at the coordinates $[0.72,0.5]$, based on the midpoint function (circle point in Figure 4-left). Following the procedure, described in the previous chapter, the clustering of Figure 4-right was derived. In this diagram, the three clusters are represented with different colours and the final location of the weights can be observed. The output layer of this network was a 3 x 100 matrix indicating which cluster-neuron each element belonged to.

\subsection{DSM clustering}

The method begins with the assumption that an $n$-dimensional clustering problem has to be solved, where $n$ the number of the elements of the product. By considering the 
DSM of a product as the matrix, which contains the lengths of $\boldsymbol{v}_{\boldsymbol{i}}$ at each dimension, the $n$ vectors of $n$ dimensions will derive as input for the network:

$$
\left[\begin{array}{llll}
v_{1} & v_{2} & \cdots & v_{i}
\end{array}\right]=\left[\begin{array}{|l|l|l|l|l|l}
\hline 1 & 1 & 1 & & 1 & \\
\hline 1 & 1 & & & 1 & \\
\hline 1 & & & & & 1 \\
\hline & & & & & \\
\hline 1 & 1 & & & 1 & \\
\hline & & 1 & 1 & & 1 \\
\hline
\end{array}\right]\left[\begin{array}{c}
\vec{\imath} \\
\vec{j} \\
\vec{k} \\
\cdot \\
\cdot
\end{array}\right]
$$

It should be remarked, that the values of the diagonal cells are filled with the value " 1 ", in order for the algorithm to include the $i^{\text {th }}$ element in the cluster that contains the elements interacting with it. This vector representation will enable the introduction of each element's interactions with the Neural Network.

Following this vector representation of the DSM, the SONN is executed as described in the previous chapter. A novelty introduced through the utilization of this algorithm for DSM clustering, is that with the help of bias the design engineer is able to preselect the number of clusters that will be deriving. Furthermore, considering that each time that the network is run, it produces different clustering alternatives, a multi-criteria decision making approach is employed in order to identify the optimum clustering solution from the set of alternatives generated (Chryssolouris 2006). In Table 2, $A$ is the clustering alternative, index $k$ is the number of the alternatives, generated by the network, $S_{\text {in }}$ is the sum of the cells having a zero value inside the clusters, $S_{\text {out }}$ is the sum of the interactions outside the clusters, $W_{l}$ and $W_{2}$ are weight factors and finally, $C E$ stands for the clustering efficiency index and derives from the equation that follows. The bigger the $C E$ is the more efficient is the clustering.

$$
\mathrm{CE}=1 /\left(\mathrm{W}_{1} * \mathrm{~S}_{\mathrm{in}}+\mathrm{W}_{2} * \mathrm{~S}_{\mathrm{out}}\right)
$$


The values of the weights $W_{l}$ and $W_{2}$ are chosen depending on the clustering outcome expected by the designer. A greater $W_{l}$ would result in denser clusters and a greater $W_{2}$ in fewer external interactions. Since these coefficients are interdependent, they should meet the following equation:

$$
W_{1}+W_{2}=1
$$

\section{Case study}

A car's Body in White (BiW) was selected as a case study in order for the proposed algorithm to be applied and evaluated. The BiW DSM presented in the work of Pandremenos et al. (2009) was utilized. It consists of 38 parts and 108 interactions among them (Figure 5). In order to assess the clustering efficiency the $M P$ was calculated to have the value of 0.89 . Since this value reveals a quite modular architecture, the clustering is not expected to be very efficient. However, the automotive industry seems to be benefiting from it and thus, it is applied to BiWs. Deleted: s Deleted: it in From the clustered BiWs, platform modules may occur and through them different car variants can be generated. Most of the times, the clustering is performed empirically, since it is based on the knowledge and experience of design engineers. The BiW utilized in this study is empirically clustered into five modules, as shown in Figure 6. The same DSM has been clustered by the method introduced in this paper. The algorithm was set to produce five modules (the same with the empirical clustering and was run for 50 times. Therefore, $k=50$ different DSM clustering alternatives were obtained and evaluated with the $C E$ index. For the calculation of $C E$, a 0.5 value was selected for $W_{l}$ and $W_{2}$. The results are shown in Figure 8 . It can be easily observed that all the $C E$ values having derived from the different runs of the Neural Network, are much higher than the $C E$ of the empirical clustering. The 
maximum $C E$ is derived from the $28^{\text {th }}$ run and it is equal to 0.009901 . The clustered DSM having resulted from this run, is shown in Figure 7. It should be also pointed out that the clustering of the case study, derived within 30 seconds only, with the use of the MATLAB R2009b software installed in a PC, having an "Intel Core 2 Duo SP9400/2.4GHz/1066MHz/6MB" processor and 4 GB RAM.

\section{Conclusions}

The clustering efficiency study has revealed that the most efficient clustering is achieved when dealing with products of a combined design architecture. Moreover, the Neural Network algorithm developed proved to have important novelties:

- Easy and fast to be formulated for a successful DSM clustering.

- The number of modules produced can be either predefined by the operator, with the help of the Bias, or determined by the network itself (without the use of the Bias).

- The efficiency of the clustering was measured with an index which took into consideration the number of interactions both within and outside the clusters.

The method was successfully applied to a real case study, a car BiW, and five modules were obtained. Similarities, in front and rear end modules are observed between the clustering of the proposed method and the empirical clustering. From Figure 7, it can be easily observed that the algorithmic clustering is still not efficient enough (as it was expected from the calculation of the $M P$ ). However, the evaluation of the two approaches with the help of the $C E$ index has shown a more efficient clustering for the case of the algorithm (Figure 8). The feasibility of this clustering architecture though, is an issue that needs to be further investigated. Several aspects, such as the integrity of the modules during assembly, the way product variation can be derived from such modules' split up, the rigidity of the final product and others, may affect the feasibility of a clustering architecture. The approach, at its current 
condition, can be used by design engineers as a tool for the derivation of product clustering suggestions, during the new product family/platform design. Moreover, the approach is also valid for everything that can be represented through a DSM (e.g. software, project teams etc.).

In future work, more case studies will be investigated and the criteria for the clustering assessment will be enriched so as to take into account more aspects. The integration of the $C E$ index into the network's architecture will also be considered in such a way so as to produce directly the optimum clustering solution (reinforcement learning). Additionally, a quantitative comparison of the $M P$ with the $C E$ will be tried and the capability of the network to produce bus clusters will be examined. Finally, this approach will be compared with other existing algorithms.

\section{Acknowledgement}

The work was partially supported by the EU funded project: FP6-2004-NMP-NI-4-026621, MultiFUnctional maTerials and related prodUction technologies integRated into the Automotive industry of the future-FUTURA".

\section{References}

Browning, T.R., 2001. Applying the Design Structure Matrix to System Decomposition and Integration Problems: A review and new directions. IEEE Transactions on engineering management, 48 (3), 292-306.

Chryssolouris, G., 2006. Manufacturing systems: Theory and Practice. 2nd ed. New York: Springer.

Ericsson, A. and Erixon, G., 1999, Controlling Design Variants, Dearborn, Michigan: Society of Manufacturing Engineers.

Fernandez, C.I.G., 1998. Integration analysis of product architecture to support effective team co-location. Dissertation. Massachusetts Institute of Technology.

Gu, P., Hashemian, M. and Nee, A.Y.C., 2004, Adaptable design, Annals of CIRP, 53 (2), $539-557$.

Idicula, J., 1995. Planning for concurrent engineering. Dissertation. Nanyang Technological University.

Kohonen, T., 2001. Self-Organizing Maps. 3rd ed. Heidelberg: Springer.

Mueller, C., 2004. Sparse Matrix Reordering Algorithms for Cluster Identification. For I532, Machine Learning in Bioinformatics. 
Oliveira, S., Ribeiro, J.F.F. and Seok, S.C., 2009. A spectral clustering algorithm for manufacturing cell formation. Computers and Industrial Engineering, 57 (3), 1008-1014.

Pandremenos, J., Chatzikomis, C. and Chryssolouris, G., 2009. On the Quantification of Interface Design Architectures. Asian International Journal of Science and Technology in Production and Manufacturing, 2 (3), 41-48.

Pugh, S., 1991, Total design: integrated methods for successful product engineering, New York: Prentice Hall.

Rogers, J.L., Korte, J.J. and Bilardo Jr., V.J., 2006. Development of a Genetic Algorithm to Automate Clustering of a Dependency Structure Matrix. NASA Technical Reports Server (NTRS).

Sangal, N. Et al., 2005. Using Dependency Models to Manage Complex Software Architecture. OOPSLA' 05, 167-176.

Simpson, T., Siddique, Z. and Jiao, J., 2006, Platform-Based Product family Development, Product Platform and Product Family Design: Methods and Applications, New York: Springer, 1-15.

Suh, N.P., 1990, The principles of design, New York: Oxford University Press.

Thebeau, R.E., 2001. Knowledge management of system interfaces and interactions for product development processes. Dissertation. Massachusetts Institute of Technology.

Ulrich, K.T., 1995. The role of product architecture in the manufacturing firm. Research Policy, 24, 419-440.

Ulrich, K.T. and Eppinger, S.D., 2003. Product Design and Development. 3rd ed. New York: McGraw-Hill.

Vesanto, J. and Alhoniemi, E., 2000. Clustering of Self-Organizing Map. IEEE transactions on Neural Networks, 11 (3), 586-600.

Yassine, A. and Braha, D., 2003. Complex Concurrent Engineering and the Design Structure Matrix Method. Concurrent Engineering: Research and Applications, 11 (3), 165-176.

Yassine, A., 2004. An Introduction to Modeling and Analyzing Complex Product Development Processes Using the Design Structure Matrix (DSM) Method. Italian Management Review, 9, 72-78.

Yu, T.-L., Yassine, A.A. and Goldberg, D.E., 2003. A genetic algorithm for developing modular product architectures. Proceedings of DETC' 03.

Yu, T.-L., Yassine, A.A. and Goldberg, D.E., 2007. An information theoretic method for developing modular architectures using genetic algorithms. Res Eng Design, 18, 91-109. 


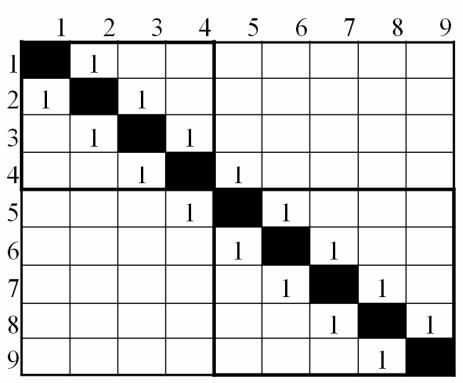

a. Modular design architecture

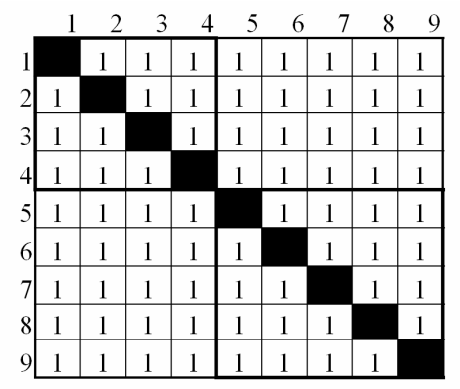

b. Integral design architecture

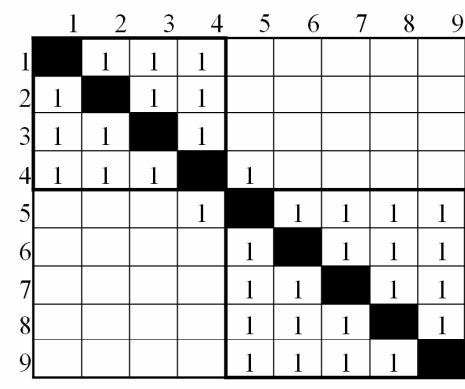

\section{c. Combined design} architecture

Figure 1. Clustering on Modular, Integral and Combined design architectures.

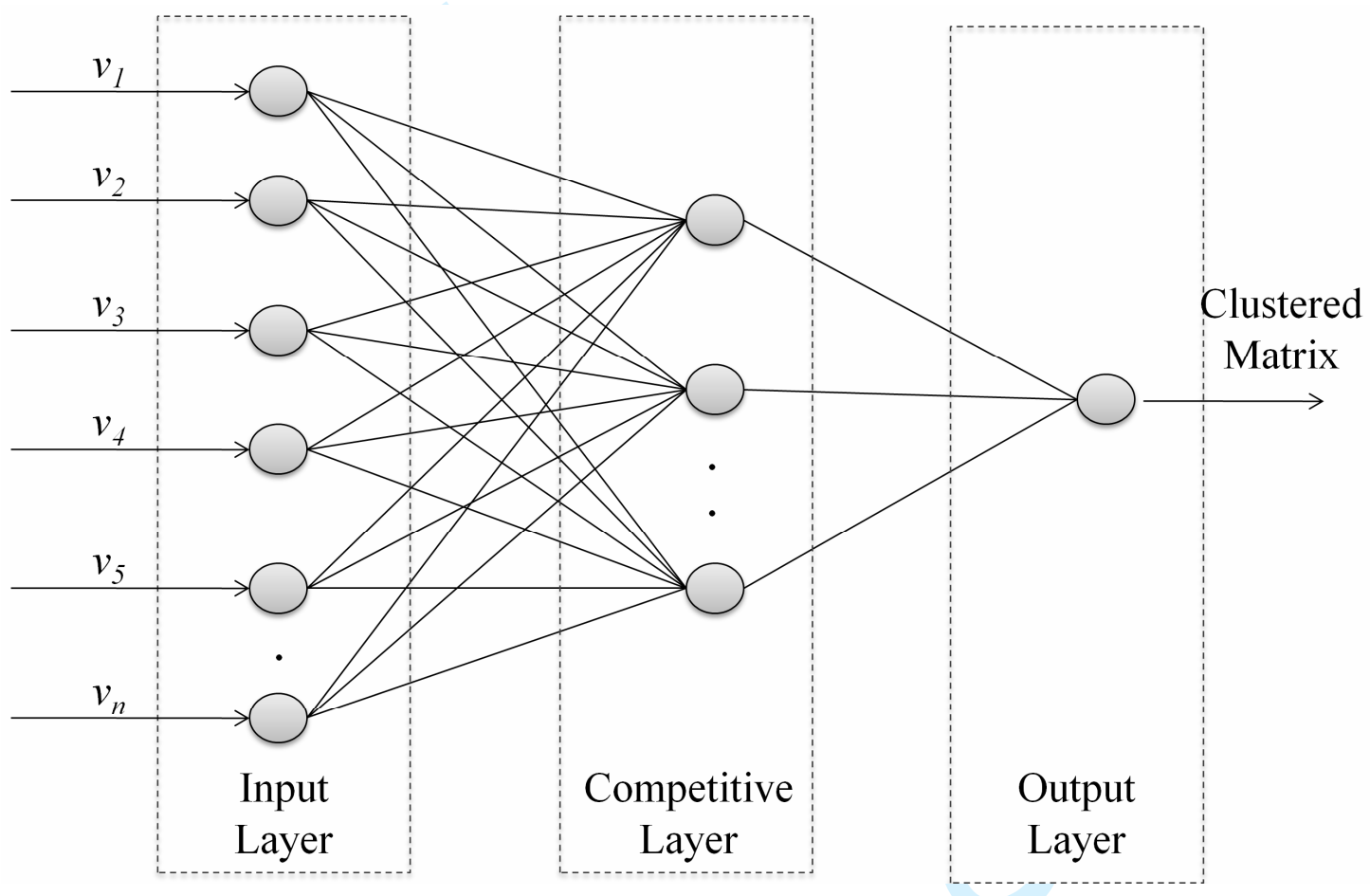

Figure 2. Neural Network architecture. 


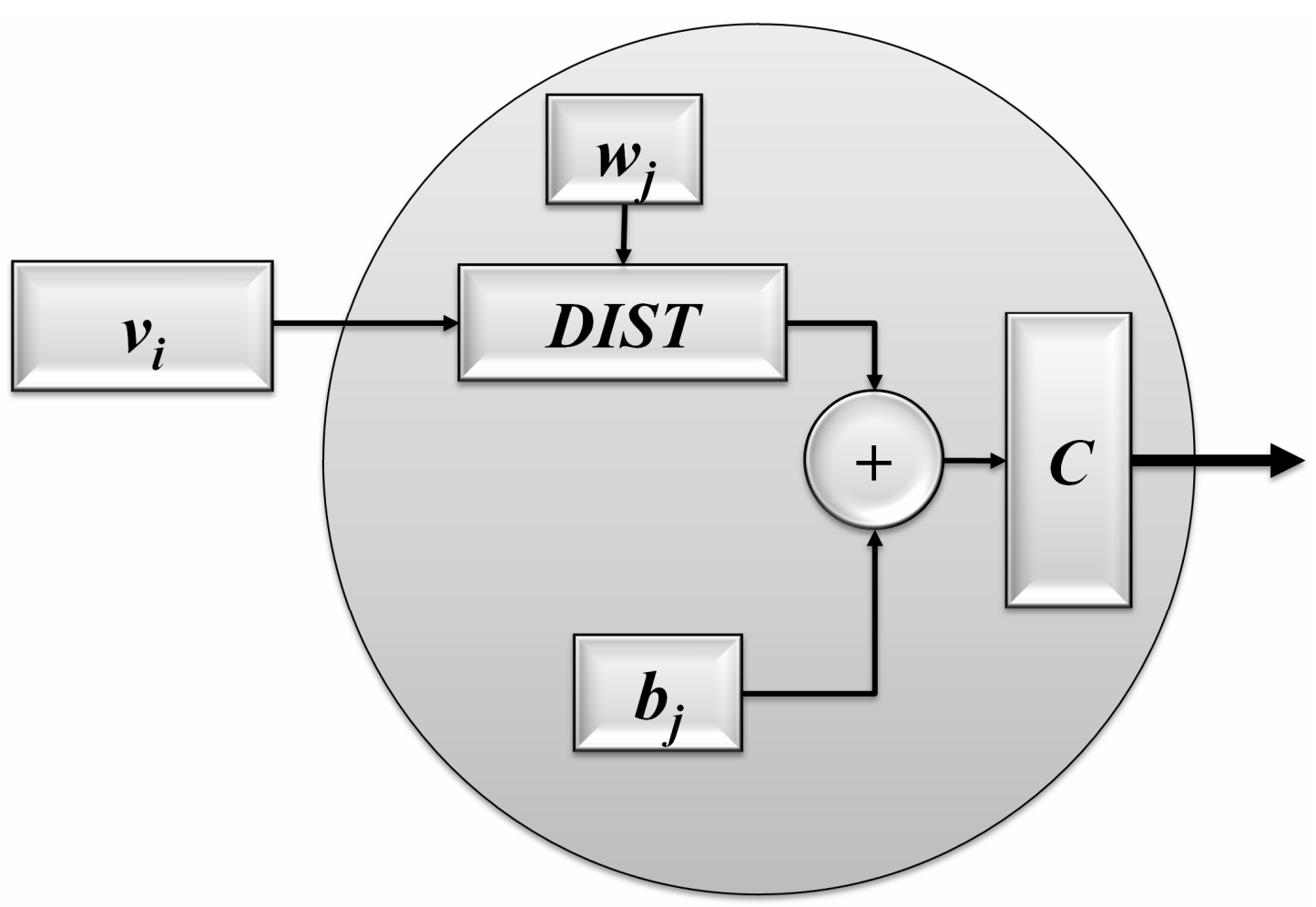

Figure 3. Neuron architecture in competitive layer.
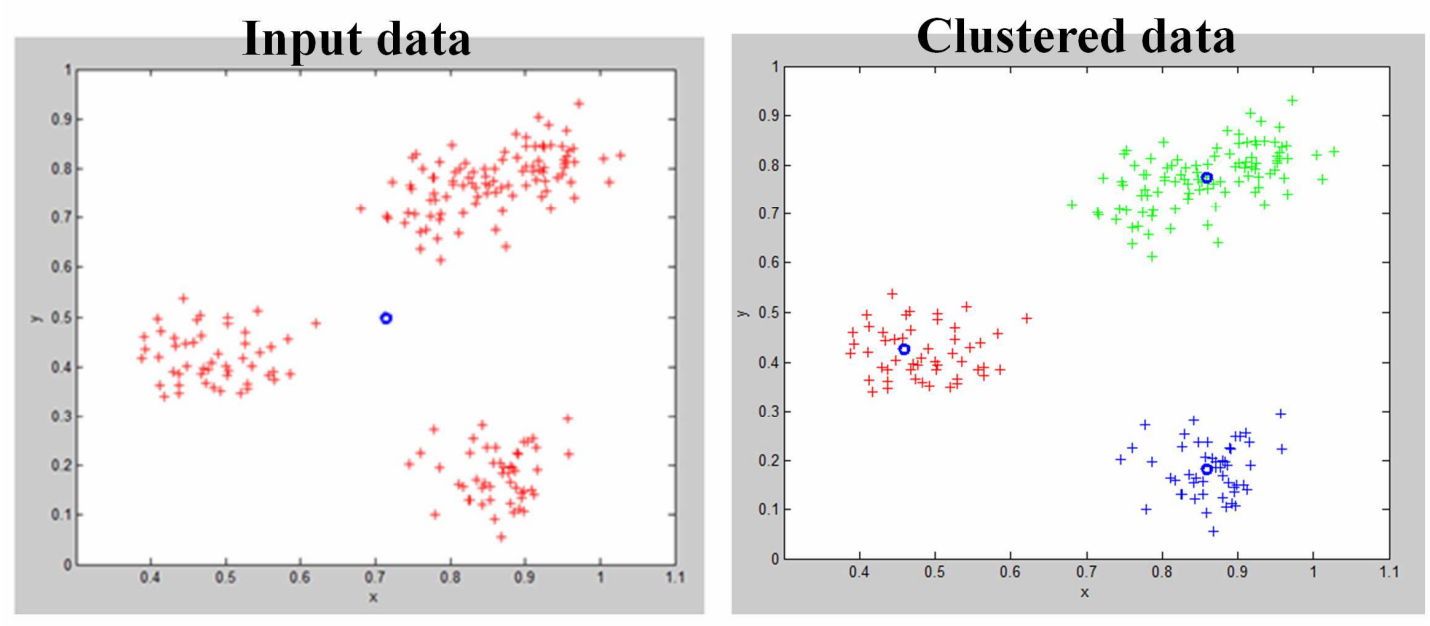

Figure 4. Input (left) and clustered 2-dimensional data (right). 


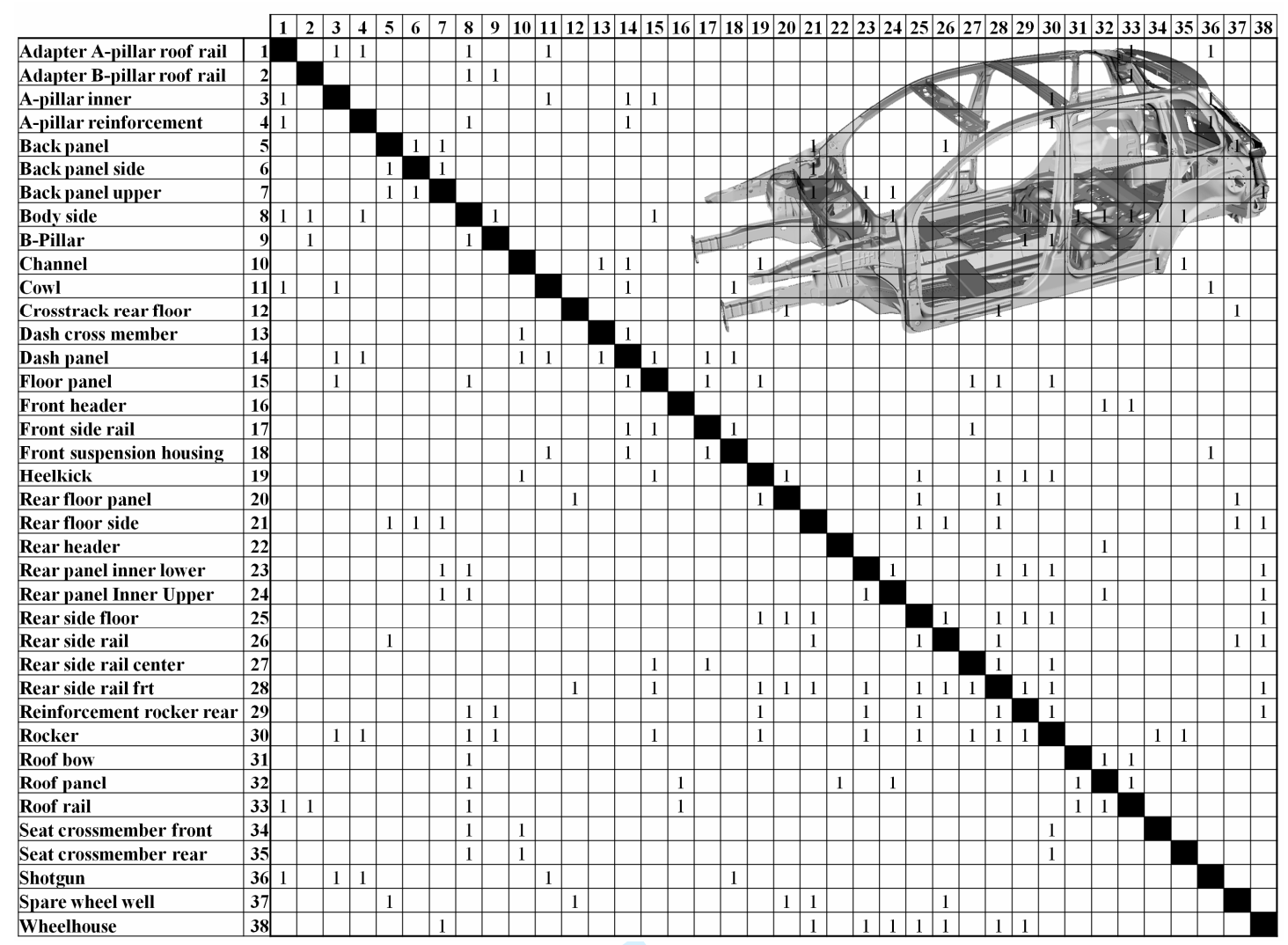

Figure 5. BiW DSM.

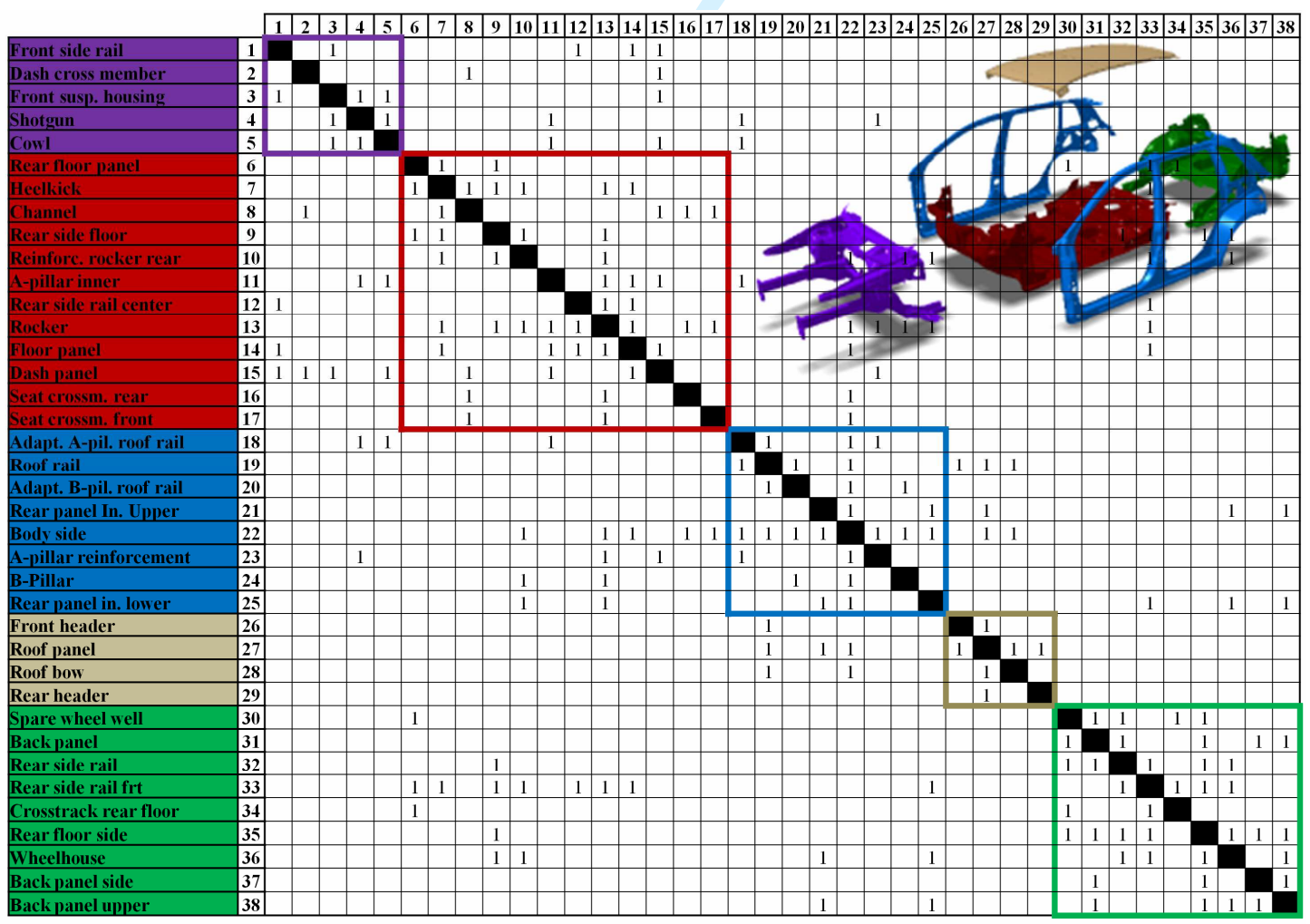

Figure 6. BiW DSM empirical clustering. 


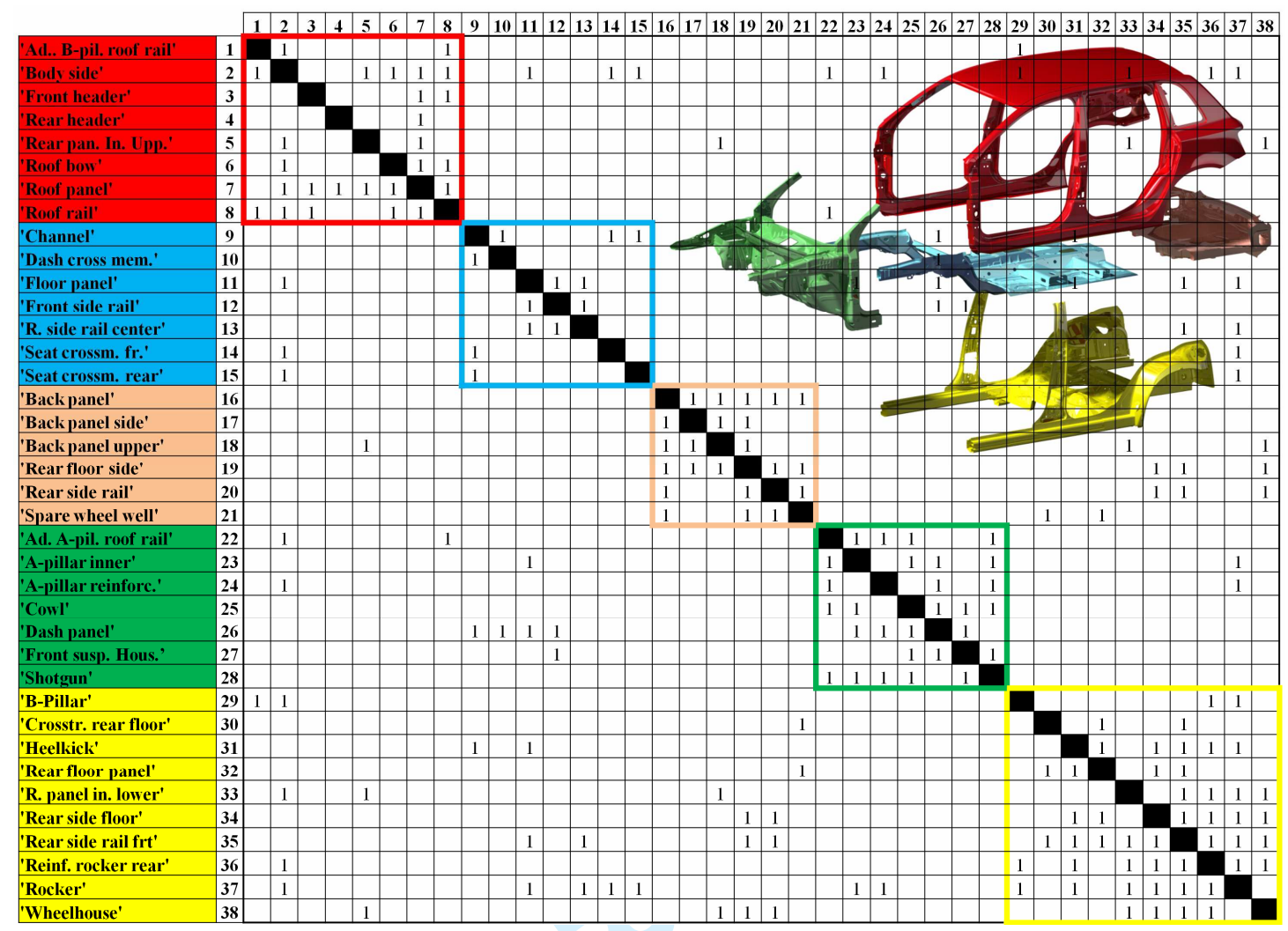

Figure 7. BiW DSM algorithm based clustering.

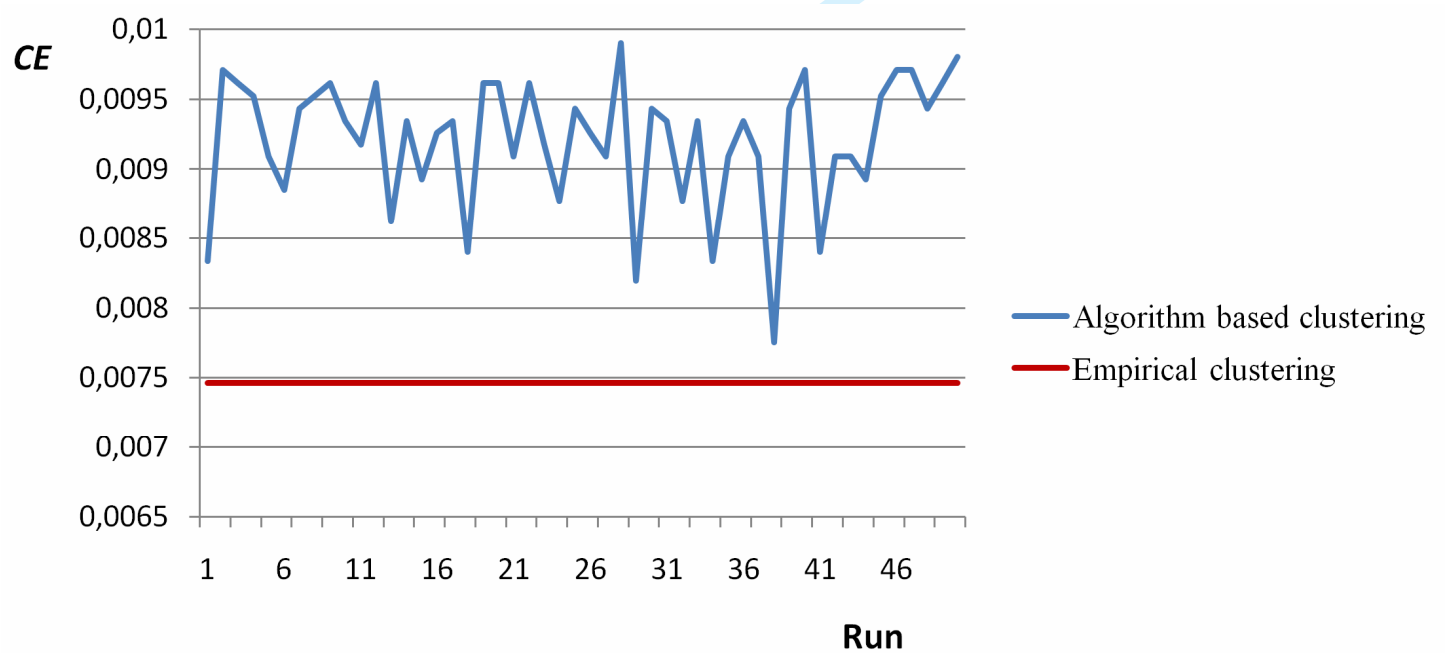

Figure 8. CE for empirical and algorithm based clustering of BiW DSM. 


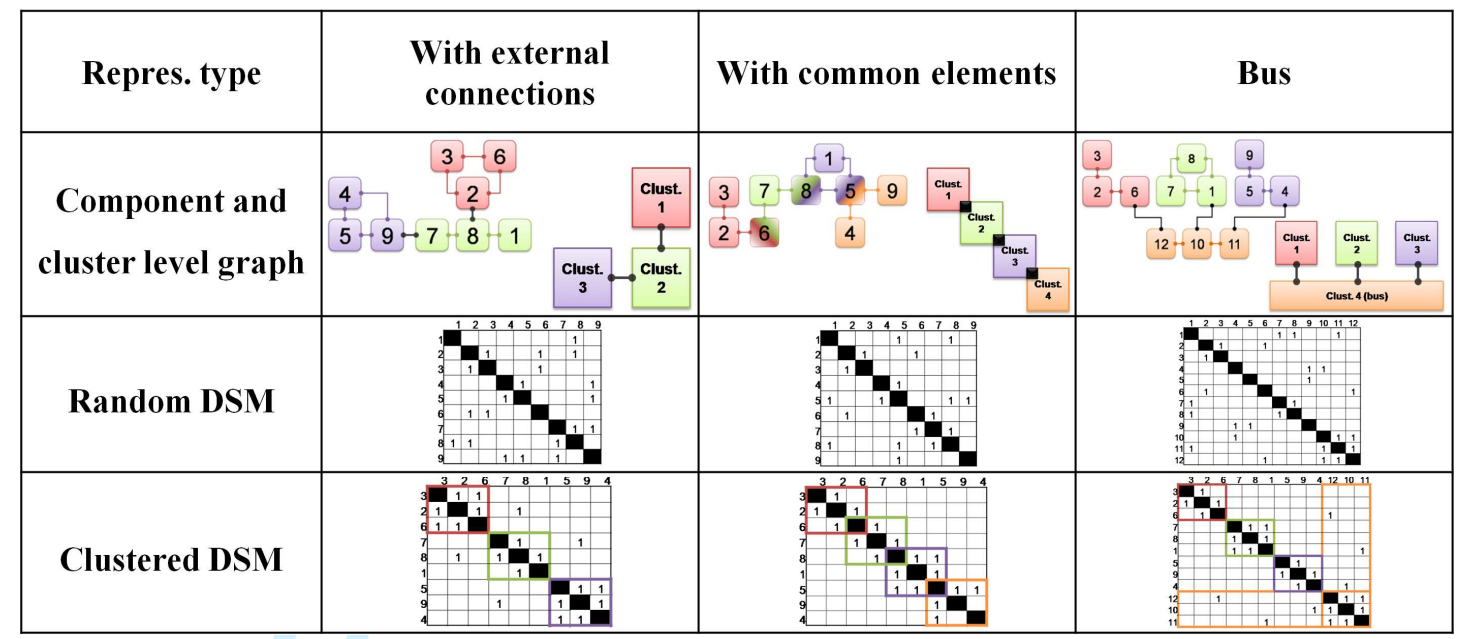

Table 1. Clustering types.

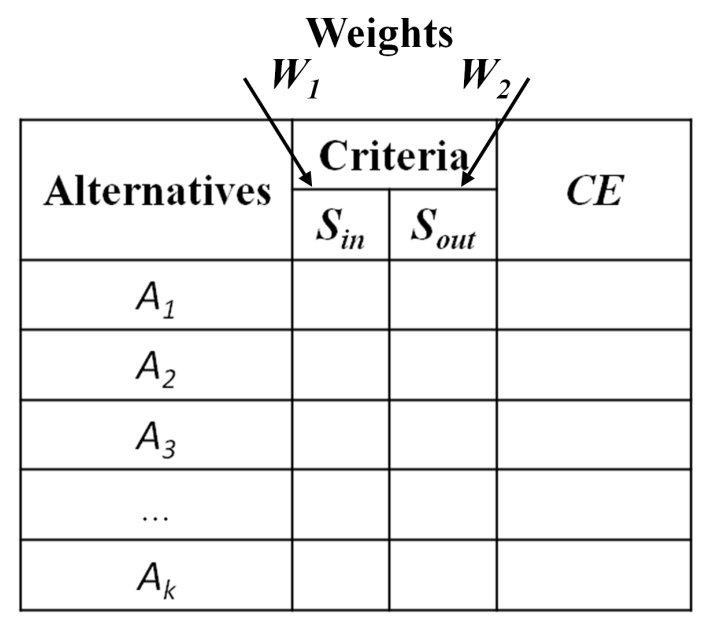

Table 2. Multi-criteria decision making table. 